\section{A) Check for updates}

Cite this: Dalton Trans., 2018, 47 283

Received 12th September 2017, Accepted 15th November 2017 DOI: $10.1039 / \mathrm{c} 7 \mathrm{dt} 03390 \mathrm{~g}$ rsc.li/dalton

\title{
Photoresponsive ruthenium-containing polymers: potential polymeric metallodrugs for anticancer phototherapy
}

\author{
Wen Sun, Xiaolong Zeng and Si Wu (D) *
}

This Frontier presents the recent development of photoresponsive Ru-containing polymers for cancer treatment. These novel Ru-containing polymers are prepared by introducing photoresponsive Ru complexes into polymers. Based on their chemical structures in aqueous solutions, these polymers can selfassemble into different nanostructures. The self-assembled nanostructures can circulate in the blood stream, accumulate at tumor tissue, and can be taken up by tumor cells. Red light, which can penetrate into tissue deeply, can induce the photodissociation of these polymers and sensitize singlet oxygen $\left({ }^{1} \mathrm{O}_{2}\right)$ generation. Both dissociated $\mathrm{Ru}$ complexes and generated ${ }^{1} \mathrm{O}_{2}$ can inhibit the growth of tumor cells. Photoresponsive Ru-containing polymers provide a new platform for combined photodynamic therapy and photoactivated chemotherapy. The design strategies, self-assembly, photoresponsiveness, and anticancer effects of these polymers are introduced. Some remaining challenges for Ru-containing polymers for phototherapy are discussed.

\section{Introduction}

The design of new drugs for cancer treatment has been a major aspect in fundamental research. ${ }^{1,2}$ Metallodrugs play a vital role in this field. It is well known that the platinum complex, cisplatin, is the first US Food and Drug Administration (FDA) approved anticancer drug, which has been effectively used in clinics. ${ }^{2-4}$ However, cisplatin has side effects and suffers from drug resistance, which motivated researchers to develop new metallodrugs. ${ }^{5}$ As analogues of cisplatin, ruthenium $(\mathrm{Ru})$ complexes are one of the good alternatives. $^{1,2}$ To date, a large number of $\mathrm{Ru}$ complexes, which exhibit anticancer activities, have been designed., ${ }^{1,2,6}$ After uptake by cancer cells, Ru complexes can bind with DNA, which effectively block DNA and RNA synthesis, leading to programmed cell death. ${ }^{6-8}$ The increasing interest in $\mathrm{Ru}$ complexes is also due to their appealing photoresponsive properties. $^{2,9-12}$ Some $\mathrm{Ru}$ complexes can generate singlet oxygen under light irradiation, which enables them to be photosensitizers for photodynamic therapy (PDT). ${ }^{10,13-15}$ In addition, light can uncage toxic Ru species or ligands from some $\mathrm{Ru}$ complexes for photoactivated chemotherapy (PACT). ${ }^{9,10}$ Therefore, some Ru complexes may be applied to combined PDT and PACT. We call them "phototherapy" in general in this Frontier.

Max Planck Institute for Polymer Research, Ackermannweg 10, 55128 Mainz, Germany.E-mail:wusi@mpip-mainz.mpg.de
The use of photoresponsive $\mathrm{Ru}$ complexes for anticancer phototherapy has attracted increasing attention because combined PDT and PACT can improve therapeutic efficiency. ${ }^{1,2,9,10,13-15}$ The processes of using Ru complexes for phototherapy are similar to those of other photosensitive anticancer agents, such as photosensitizers or photoactivated (pro)drugs for PDT or PACT. ${ }^{16,17}$ First, photosensitive anticancer agents (e.g., Ru complexes) should be injected into the blood vessel. Then, the agents with appropriate properties could accumulate at the tumor site through blood circulation and internalize into cancer cells. Light irradiation at the tumor site will activate the $\mathrm{Ru}$ complexes to generate toxic species, and consequently, inhibit tumor growth.

\section{Deep-tissue photoactivation of $\mathrm{Ru}$ complexes with red light}

Light penetration into tissue is one of the preconditions for phototherapy. ${ }^{18}$ Many photoresponsive compounds, such as, o-nitrobenzyl, pyrene, and coumarin, have been studied and proposed for phototherapy. ${ }^{18,19}$ However, they are responsive to UV light or short wavelength visible light, which cannot penetrate deeply into tissue. In comparison, photoresponsive $\mathrm{Ru}$ complexes are promising for deep-tissue phototherapy because some of them are sensitive to red or near infrared (NIR) light in the "therapeutic window" (e.g., 650-900 nm). ${ }^{2,16,20}$ For example, our group synthesized four photoresponsive 
(a)
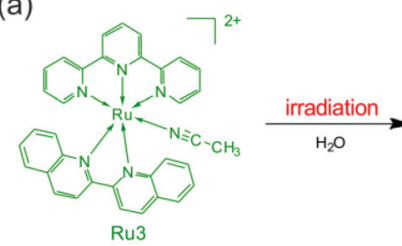

$\mathrm{H}_{2} \mathrm{O}$
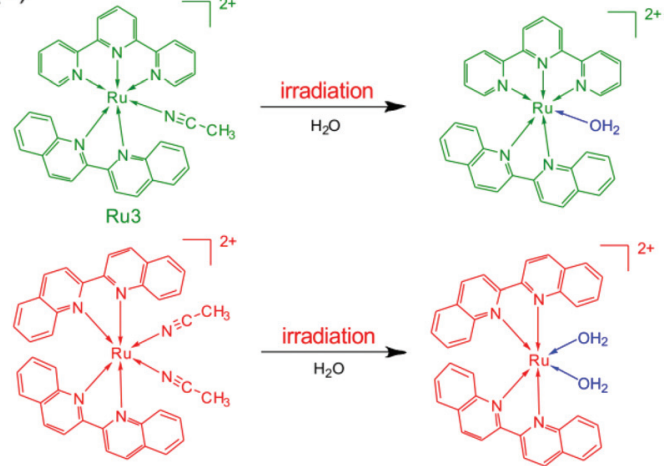

Ru4
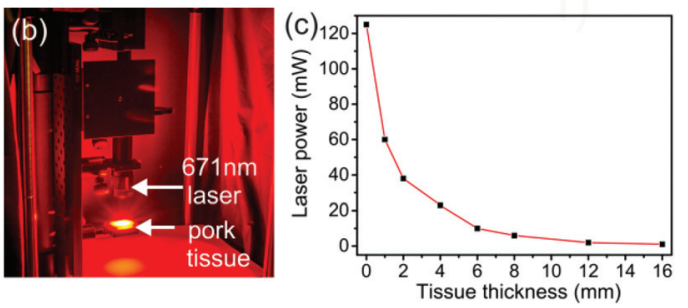

Fig. 1 (a) Photoreactions of Ru3 and Ru4 under red light irradiation. (b) Photograph showing the laser (671 nm, $125 \mathrm{~mW}$ ) passing through pork tissue. A laser spot can be observed after passing through the pork tissue. (c) Laser power after the laser (671 nm and $125 \mathrm{~mW}$ ) passed through the pork tissue with different thicknesses. Reproduced with permission from ref. 21. Copyright 2017 Wiley-VCH Verlag GmbH \& Co. KGaA.

$\mathrm{Ru}$ complexes and studied the photoactivation of these complexes in deep tissue. ${ }^{21}$ Two of them (Ru3 and Ru4) have absorption tails in the "therapeutic window" and can be activated using red light (Fig. 1a). We demonstrated that $671 \mathrm{~nm}$ red light can activate Ru3 and Ru4 after passing through a 16 mm-thick tissue (Fig. 1b and c). Additionally, activated Ru4 caused the inhibition of cancer cells. These results suggested that red-light-responsive $\mathrm{Ru}$ complexes (e.g., Ru3 and Ru4) are promising for deep-tissue phototherapy. Photoresponsive $\mathrm{Ru}$ complexes are considered as promising agents for phototherapy and have been widely used in vitro. ${ }^{17}$ However, photoresponsive $\mathrm{Ru}$ complexes are problematic for in vivo applications. First, $\mathrm{Ru}$ complexes display poor bioavailability for cancer treatment. They can be rapidly cleared from the bloodstream, leading to inefficient accumulation at tumor sites. ${ }^{22}$ This problem could result in low therapeutic efficiency. Second, similar to other anticancer agents, $\mathrm{Ru}$ complexes may also cause serious toxic side effects on healthy tissues. ${ }^{1}$ Thus, improving the therapeutic efficiency of photoresponsive Ru complexes and reducing their side effects are currently two challenging problems.

\section{Photoresponsive Ru-containing polymers}

To overcome the above-mentioned problems of low molecular weight $\mathrm{Ru}$ complexes, we designed Ru-containing block co- polymers (BCPs) that could self-assemble into nanostructures in aqueous solutions. ${ }^{23,24}$ Photoresponsive $\mathrm{Ru}$ complexes are building blocks and functional moieties of the BCPs, which can be activated in tumor cells with light. Compared to low molecular weight $\mathrm{Ru}$ complexes, Ru-containing BCPs with proper self-assembled nanostructures and surface chemistry have longer blood circulation. In particular, nanoparticles with sizes of 10-200 nm show effective tumor accumulation through the enhanced permeability and retention (EPR) effect. $^{25,26}$ Thus, self-assembled BCP nanoparticles may improve the bioavailability of photoresponsive $\mathrm{Ru}$ complexes and reduce their toxic side effects.

Our group, for the first time, synthesized red-light-responsive Ru-containing BCPs and demonstrated their potential applications in phototherapy. Three side-chain Ru-containing BCPs (P1-P3) with different molecular weights were prepared (Fig. 2). ${ }^{23}$ Each BCP contained a hydrophilic and biocompatible poly(ethylene glycol) (PEG) block and a hydrophobic Rucontaining block. Depending on the molecular weight, the BCPs assembled into micelles, vesicles, and large compound micelles. All of the nanostructures could be taken up by cancer cells and the micelles showed the highest cellular uptake efficiency compared to the other two. The red-light irradiation of the BCPs released the anticancer Ru complexes and generated reactive ${ }^{1} \mathrm{O}_{2}$ in cancer cells. The released anticancer $\mathrm{Ru}$ complex can be used for PACT and ${ }^{1} \mathrm{O}_{2}$ can be used for PDT. The combined PDT and PACT showed enhanced anticancer activity. The cell viability decreased to $24 \%, 75 \%$ and $80 \%$

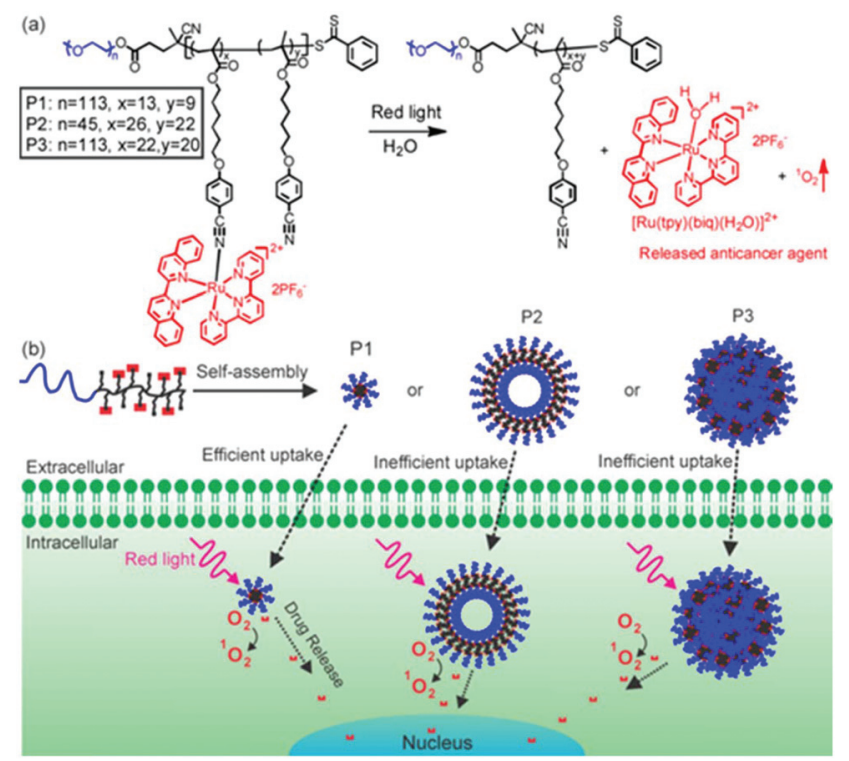

Fig. 2 (a) Chemical structures of side-chain Ru-containing BCPs. Red light irradiation releases the Ru complex and generates singlet oxygen $\left({ }^{1} \mathrm{O}_{2}\right) . P 1, P 2$ and P3 are BCPs with different molecular weights. (b) Schematic illustration of the self-assembly of Ru-containing BCPs. P1, P2 and P3 self-assemble into micelles, hollow spheres and large compound micelles, which exhibit morphology-dependent cellular uptake and anticancer performance. Reproduced with permission from ref. 23. Copyright 2016 Wiley-VCH Verlag GmbH \& Co. KGaA. 


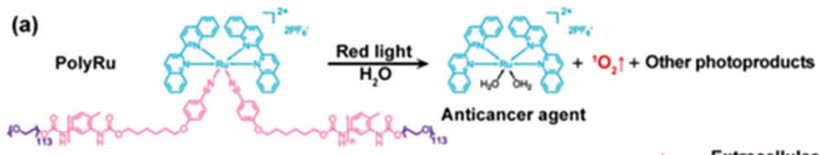

(b)
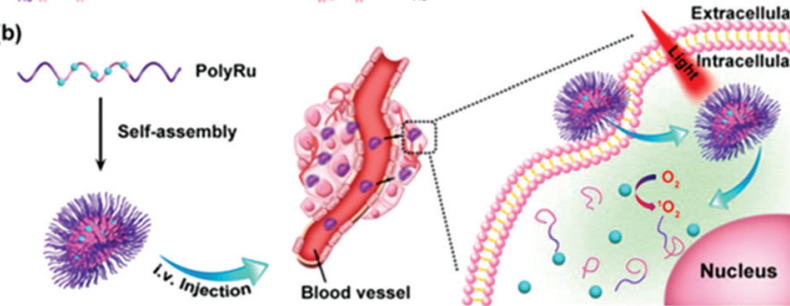

Fig. 3 (a) Chemical structure of the main-chain Ru-containing BCP (PolyRu). Red light induces the degradation of PolyRu to generate the anticancer complex and ${ }^{1} \mathrm{O}_{2}$. (b) Schematic illustration of self-assembly and phototherapy using PolyRu. Reproduced with permission from ref. 24. Copyright 2017 Wiley-VCH Verlag GmbH \& Co. KGaA.

when cells were incubated with micelles, vesicles and large compounds, followed by $656 \mathrm{~nm}$ red light irradiation. The anticancer performance of micelles was better than those of vesicles and large compound micelles due to their more efficient cellular uptake. Moreover, under the dark conditions, there was no cleavage of the Ru complex from the BCP assemblies, suggesting that no side effect would occur in a normal microenvironment.

We also introduced Ru complexes into the main-chain of a BCP (Fig. 3). ${ }^{24}$ PolyRu is an ABA-type triblock copolymer that contains a hydrophobic and photolytic Ru-containing block and two hydrophilic and biocompatible PEG blocks (Fig. 3). The weight fraction of the Ru-containing block in PolyRu was more than 50\%, demonstrating an efficient drug-loading capacity of the BCP. In an aqueous solution, PolyRu selfassembled into nanoparticles with an average diameter of $180 \mathrm{~nm}$. The PolyRu nanoparticles could be effectively taken up by the cancer cells including HeLa, PC3, and HepG2. The photodegradation of PolyRu induced by $656 \mathrm{~nm}$ red light facilitated the release of anticancer $\mathrm{Ru}$ complexes, and consequently, the proliferation of cancer cells was efficiently inhibited. More importantly, in vivo experiments in a mouse model demonstrated that Ru-containing BCPs could accumulate at tumor sites and inhibit the growth of tumor under light irradiation. In addition, the nanoparticles did not cause any pathological tissue damage/abnormality during the treatment. Thus, phototherapy using Ru-containing BCPs eliminated systemic toxicity caused by Ru complexes in vivo.

\section{Conclusions and outlook}

In conclusion, photoresponsive Ru-containing BCPs have been developed for anticancer phototherapy. These BCPs selfassembled into nanoparticles which could circulate in the blood stream, accumulate at tumor tissue, and inhibit the growth of cancer cells under red-light irradiation. Both the released toxic $\mathrm{Ru}$ complexes and generated ${ }^{1} \mathrm{O}_{2}$ exhibit the anticancer effect. More importantly, Ru-containing nanoparticles can improve the bioavailability of $\mathrm{Ru}$ complexes and reduce their toxic side effects in vivo. The design strategies described here can be used to develop new and improved $\mathrm{Ru}-$ containing polymers for anticancer phototherapy. The studies using photoresponsive Ru-containing polymers for biomedical applications have just started. There are some open questions and challenges regarding their synthesis, characterization, and applications. This field still awaits more exploration.

First, from the chemistry point of view, finding ways to synthesize photoresponsive Ru-containing polymers with controlled molecular weights and architecture is a challenge. In our work, we either grafted Ru complexes to polymers (Fig. 2) or used polycondensation to polymerize Ru-containing monomers (Fig. 3). The "graft-to" method can only functionalize a part of the repeat units on the polymers. Polycondensation is difficult to control the molecular weight. Photo-responsive Rucontaining polymers, which are directly polymerized using controlled polymerization, have not been demonstrated.

Second, from the characterization point of view, it is difficult to analyze photoresponsive Ru-containing polymers using standard polymer analytical methods such as gel permeation chromatography (GPC) and static light scattering (SLS). The presence of charged moieties in such polymers may result in unreliable molecular weight characterization via GPC due to their interaction with the GPC column material. ${ }^{27}$ The measurement using SLS is also difficult because the light irradiation of the sample with an inappropriate wavelength may cause the dissociation of the polymers. Suitable characterization methods to analyze such polymers are expected to be conducted.

Third, from the therapeutic point of view, the anticancer efficiency of responsive Ru complexes needs further improvement. The $\mathrm{Ru}$ complexes used in the reported systems only demonstrated acceptable toxicity, which results in the moderate anticancer efficiency of these Ru-containing polymers. Developing polymers using high toxicity photoresponsive $\mathrm{Ru}$ complexes will help us improve the therapeutic efficacy of $\mathrm{Ru}$ containing polymers.

Fourth, the activation wavelength of Ru-containing polymers needs to be further red-shifted. The best activation wavelength is at the NIR region. However, the absorption tails but not the peaks of the reported compounds just reach the NIR region. Although the two-photon process ${ }^{28,29}$ and upconversion-assisted photochemistry ${ }^{18,30-38}$ can activate $\mathrm{Ru}$ complexes using NIR light, both methods are based on inefficient nonlinear optical processes. Two-photon absorption requires unsafe high-intensity laser excitation and only occurs at the focus of pulsed lasers. Thus, two-photon absorption is not suited for phototherapy applications. Upconversion-assisted photoactivation often requires a laser intensity of at least several hundred $\mathrm{mW} \mathrm{cm}^{-2}$. Because light intensity decreases dramatically after passing through tissue (Fig. 1c), upconversion is problematic for deep-tissue applications. Thus, the direct red-shift of the activation wavelength of Ru-containing polymers to the NIR region is more promising for deep-tissue 
applications. For example, the use of fluorinated aromatic rings or compounds with high aromaticity may help to red shift the activation wavelength. Such Ru-containing polymers could be sensitive to low-intensity NIR light, which is suited for phototherapy in real applications.

\section{Conflicts of interest}

There are no conflicts to declare.

\section{Acknowledgements}

W. S. and X. Z. were supported by the CSC program. This work was supported by the Fonds der Chemischen Industrie (FCI, No. 661548) and the Deutsche Forschungsgemeinschaft (DFG, WU 787/2-1). Open Access funding was provided by the Max Planck Society.

\section{Notes and references}

1 L. Zeng, P. Gupta, Y. Chen, E. Wang, L. Ji, H. Chao and Z. S. Chen, Chem. Soc. Rev., 2017, 46, 5771-5804.

2 C. Mari, V. Pierroz, S. Ferrari and G. Gasser, Chem. Sci., 2015, 6, 2660-2686.

3 F. S. Mackay, J. A. Woods, P. Heringová, J. Kašpárková, A. M. Pizarro, S. A. Moggach, S. Parsons, V. Brabec and P. J. Sadler, Proc. Natl. Acad. Sci. U. S. A., 2007, 104, 2074320748.

4 N. P. E. Barry and P. J. Sadler, ACS Nano, 2003, 7, 5654-5659.

5 V. Brabec and O. Novakova, Drug Resist. Updates, 2006, 9, 111-122.

6 J. D. Knoll and C. Turro, Coord. Chem. Rev., 2015, 282-283, 110-126.

7 E. Wachter, A. Zamora, D. K. Heidary, J. Ruiz and E. C. Glazer, Chem. Commun., 2016, 52, 10121-10124.

8 R. E. Goldbach, I. Rodriguez-Garcia, J. H. van Lenthe, M. A. Siegler and S. Bonnet, Chem. - Eur. J., 2011, 17, 99249929.

9 J. D. Knoll, B. A. Albani and C. Turro, Acc. Chem. Res., 2015, 48, 2280-2287.

10 J. K. White, R. H. Schmehl and C. Turro, Inorg. Chim. Acta, 2017, 454, 7-20.

11 L. Zayat, C. Calero, P. Alborés, L. Baraldo and R. Etchenique, J. Am. Chem. Soc., 2003, 125, 882-883.

12 L. Zayat, O. Filevich, L. M. Baraldo and R. Etchenique, Philos. Trans. R. Soc., A, 2013, 371, 20120330.

13 E. Wachter, D. K. Heidary, B. S. Howerton, S. Parkin and E. C. Glazer, Chem. Commun., 2012, 48, 9649-9651.

14 H. Huang, B. Yu, P. Zhang, J. Huang, Y. Chen, G. Gasser, L. Ji and H. Chao, Angew. Chem., Int. Ed., 2015, 54, 14049-14052.
15 N. W. Choi, S. S. Verbridge, R. M. Williams, J. Chen, J. Y. Kim, R. Schmehl, C. E. Farnum, W. R. Zipfel, C. Fischbach and A. D. Stroock, Biomaterials, 2012, 33, 2710-2722.

16 K. Liu, R. R. Xing, Q. L. Zou, G. H. Ma, H. Möhwald and X. H. Yan, Angew. Chem., Int. Ed., 2016, 55, 30363039.

17 M. Abbas, Q. L. Zou, S. K. Li and X. H. Yan, Adv. Mater., 2017, 29, 1605021.

18 S. Wu and H. J. Butt, Adv. Mater., 2016, 28, 1208-1226.

19 J. F. Gohy and Y. Zhao, Chem. Soc. Rev., 2013, 42, 71177129.

20 E. Wachter, B. S. Howerton, E. C. Hall, S. Parkin and E. C. Glazer, Chem. Commun., 2014, 50, 311-313.

21 W. Sun, R. Thiramanas, L. D. Slep, X. Zeng, V. Mailander and S. Wu, Chem. - Eur. J., 2017, 23, 10832-10837.

22 W. P. R. J. H. Koch, F. P. Dwyer and E. C. Gyarfas, Aust. J. Biol. Sci., 1957, 10, 342.

23 W. Sun, M. Parowatkin, W. Steffen, H.-J. Butt, V. Mailänder and S. Wu, Adv. Healthcare Mater., 2016, 5, 467-473.

24 W. Sun, S. Li, B. Haupler, J. Liu, S. Jin, W. Steffen, U. S. Schubert, H.-J. Butt, X.-J. Liang and S. Wu, Adv. Mater., 2017, 29, 1603702.

25 J. Wang, K. Liu, R. R. Xing and X. H. Yan, Chem. Soc. Rev., 2016, 45, 5589-5604.

26 Q. L. Zou, M. Abbas, L. Y. Zhao, S. K. Li, G. Z. Shen and X. H. Yan, J. Am. Chem. Soc., 2017, 139, 1921-1927.

27 M. A. R. Meier, B. G. G. Lohmeijer and U. S. Schubert, Macromol. Rapid Commun., 2003, 24, 852-857.

28 V. Nikolenko, R. Yuste, L. Zayat, L. M. Baraldo and R. Etchenique, Chem. Commun., 2005, 13, 1752-1754.

29 V. San Miguel, M. Alvarez, O. Filevich, R. Etchenique and A. del Campo, Langmuir, 2012, 28, 1217-1221.

30 Z. Chen, W. Sun, H. J. Butt and S. Wu, Chem. - Eur. J., 2015, 21, 9165-9170.

31 S. He, K. Krippes, S. Ritz, Z. Chen, A. Best, H. J. Butt, V. Mailänder and S. Wu, Chem. Commun., 2015, 51, 431434.

32 Z. Chen, S. He, H. J. Butt and S. Wu, Adv. Mater., 2015, 27, 2203-2206.

33 S. H. Askes, A. Bahreman and S. Bonnet, Angew. Chem., Int. Ed., 2014, 53, 1029-1033.

34 S. H. Askes, M. Kloz, G. Bruylants, J. T. Kennis and S. Bonnet, Phys. Chem. Chem. Phys., 2015, 17, 2738027390.

35 Z. Chen, Y. Xiong, R. Etchenique and S. Wu, Chem. Commun., 2016, 52, 13959-13962.

36 S. Wu, J. P. Blinco and C. Barner-Kowollik, Chem. - Eur. J., 2017, 23, 8325-8332.

37 S. Wu and H.-J. Butt, Phys. Chem. Chem. Phys., 2017, 19, 23585-23596.

38 Z. Chen, R. Thiramanas, M. Schwendy, C. Xie, S. H. Parekh, V. Mailänder and S. Wu, Small, 2017, 1700997. 